\title{
Electroencephalogram and somatosensory evoked potential evaluation for good and poor neurological prognosis after cardiac arrest: a prospective multicenter cohort trial (ProNeCA)
}

Maenia Scarpino1, Riccardo Carrai ${ }^{1,2}$, Francesco Lolli³, Giovanni Lanzo ${ }^{2}$, Maddalena Spalletti ${ }^{2}$, Daniela Audenino ${ }^{4}$, Claudio Callegarin ${ }^{5}$, Maria Grazia Celani ${ }^{6}$, Maria Lombardi ${ }^{7}$, Alfonso Marrelli ${ }^{8}$, Oriano Mecarelli ${ }^{9}$, Chiara Minardi ${ }^{10}$, Fabio Minicucci ${ }^{11}$, Luisa Motti ${ }^{12}$, Lucia Politini ${ }^{13}$, Franco Valzania ${ }^{14}$, Eugenio Vitelli ${ }^{15}$, Adriano Peris ${ }^{16}$, Aldo Amantini ${ }^{\ddagger}, 1,2$ \& Antonello Grippo*,*,1,2

${ }^{1}$ IRCCS Fondazione Don Carlo Gnocchi, Firenze

${ }^{2}$ SODc Neurofisiopatologia, Dipartimento Neuromuscolo-Scheletrico e degli Organi di Senso, AOU Careggi, Firenze

${ }^{3}$ Dipartimento di Scienze Biomediche Sperimentali e Cliniche, Università degli studi di Firenze

${ }^{4}$ SC Neurologia, Ospedale Galliera, Genova

${ }^{5}$ UO Neurologia e Neurofisiopatologia, Ospedale Santa Maria delle Croci, Ravenna

${ }^{6}$ UO Neurofisiopatologia, Ospedale Santa Maria della Misericordia, Perugia

${ }^{7}$ UO Neurologia, Ospedale San Giuseppe, Empoli

${ }^{8}$ UOC Neurofisiopatologia, Ospedale San Salvatore, L'Aquila

${ }^{9}$ UOC Neurofisiopatologia, Policlinico Umberto primo, Università La Sapienza, Roma

${ }^{10}$ UO Neurologia, Ospedale Bufalini, Cesena

${ }^{11}$ UO Neurofisiopatologia, Ospedale San Raffaele IRCCS, Milano

${ }^{12}$ UO Neurofisiopatologia Arcispedale. Santa Maria Nuova, Reggio nell'Emilia

${ }^{13}$ Ospedale Civile, Legnano

${ }^{14}$ Neurofisiopatologia Interventiva, Osp Civile di Baggiovara, Modena

${ }^{15}$ Ospedale Maggiore, Lodi

${ }^{16}$ SODc Cure intensive per il trauma ed i supporti extracorporei, Dipartimento Neuromuscolo-Scheletrico e degli Organi di Senso, AOU Careggi, Firenze

*Author for correspondence: Tel.: 0039-055-7949410; agrippo@unifi.it

$\ddagger$ Authors contributed equally

Aim: Hypoxic-ischemic-encephalopathy is a severe and frequent neurological complication of successful cardiopulmonary-resuscitation after cardiac arrest. Prognosticating neurological outcomes in patients with hypoxic-ischemic-encephalopathy is challenging and recent guidelines suggest a multimodal approach. Only few studies have analyzed the prognostic power of the association between instrumental tests and, in addition, most of them were monocentric, retrospective and evaluating only poor outcome. Methods/design: We designed a multicenter prospective cohort study to assessing the prognostic power of the association of electroencephalogram and somatosensory evoked potentials for the prediction of both poor and good neurological outcomes at different times after cardiac arrest. Discussion: The results of our study will provide a high level of evidence for the use of neurophysiological evaluation in the current clinical practice.

First draft submitted: 24 October 2018; Accepted for publication: 22 February 2019; Published online: 24 May 2019

Keywords: cardiac arrest $\bullet$ EEG $\bullet$ hypoxic ischemic encephalopathy $\bullet$ multicenter study $\bullet$ multimodal prognosis $\bullet$ neurological outcome $\bullet$ neurophysiological tests $\bullet$ ProNeCA $\bullet$ prospective study $\bullet$ SEPs

\section{Background}

Post-anoxic encephalopathy is a severe and frequent neurological complication of successful cardiopulmonary resuscitation and it is usually responsible for coma onset in patients surviving a cardiac arrest (CA) [1-3]. 
A reliable early assessment of the neurological prognosis is an important research goal because it could address CA patient management within intensive care units (ICUs). For example, knowing with certainty the patient will have a poor neurological outcome (death or unresponsive wakefulness state) can help avoid futile invasive treatments and improve the communication to relatives about the nonrecovery of consciousness and the decision to discharge patients to a nonrehabilitative long-term care unit. Even though in recent years many studies have been published regarding both poor and good long-term functional neurological outcome predictions [4-8], prognosticating outcomes after CA remains challenging.

There is evidence from the literature that neurophysiological tests, in particular somatosensory evoked potentials (SEPs) and electroencephalogram (EEG), are reliable prognostic indicators for neurological outcomes [4-7], in fact they have been included in the guidelines published in the 2015 [9] that suggest a multimodal approach for neurological prognosticating after CA. Despite these recent suggestions, to date most of the studies have analyzed a single neurophysiological test at a time $[4-6,10]$. When instead the combination of these two tests is concerned, the major limitation is represented by the study protocol: the only prospective paper reported in the literature was monocentric and evaluation of results was limited to poor neurological outcomes [8]. Thus, in order to provide a foundation for the use of EEG and SEPs as reliable prognostic tools for both poor and good outcomes in comatose patients, from an early stage (within the first $12 \mathrm{~h}$ after $\mathrm{CA}$ ), prospective multicenter trials are needed.

\section{Aim}

The principal aim of this study, 'Prognosi Neurofisiologica Coma Anossi-Ischemico' (ProNeCA) will be to evaluate the prognostic power of EEG performed in comatose patients within the first $12 \mathrm{~h}$ after CA for good outcome prediction (cerebral performance categories CPC 1-2-3) and to evaluate its prognostic power for the poor outcome prediction (CPC 4-5) when performed at 24 and $72 \mathrm{~h}$ after CA.

Moreover, we will aim to evaluate if the combination of EEG and SEPs will enable us to correctly identify a greater number of patients with both poor and good outcomes (when performed within the first $12 \mathrm{~h}$ ) and with poor outcomes (when performed after $72 \mathrm{~h}$ ) compared with the use of only a single test. In addition, we will evaluate if the concordance of EEG/SEP patterns will increase the prognostic reliability obtained with a single test.

Finally, we will aim to confirm if the prognostic power of the bilaterally absent (AA) SEP pattern for poor outcome prediction will be reliable at any time of recording after CA, and if other SEP pathological patterns will assume an analogous ominous prognostic significance.

\section{Methods}

Design

This is a prospective multicenter cohort trial. Comatose adult patients after cardiopulmonary resuscitation will be consecutively allocated in the trial. Patients will be recruited among those admitted to the ICUs of all the participating centers of the study over the course of about 24 months. Neurophysiological tests will be performed at 12 and $24 \mathrm{~h}$ after $\mathrm{CA}$ and will be repeated at $72 \mathrm{~h}$ in all the patients who, at that time, will still remain comatose.

\section{Primary outcome measure}

Neurological outcome will be the primary outcome measure and it will be assessed at 6 months after CA using CPC as follows: CPC 1, no or minor neurological deficits; CPC 2, moderate disability; CPC 3, severe disability; CPC 4, unresponsive wakefulness state and CPC 5, death. Neurological outcome will be dichotomized into 'good' (CPC 1-3) and 'poor' (CPC 4-5) outcomes.

\section{Setting}

The study will be carried out at Careggi University Hospital (Firenze), which will be the coordinating center, at San Giuseppe Hospital (Empoli), Bufalini Hospital (Cesena), Santa Maria delle Croci Hospital (Ravenna), Santa Maria Nuova Hospital (Reggio Emilia), Ospedale Civile of Baggiovara (Baggiovara-Modena), Maggiore Hospital of Lodi (Lodi-Milano), San Raffaele Hospital (Milano), Ospedale Civile of Legnano (Legnano-Milano), Policlinico Umberto Primo (Roma), Santa Maria della Misericordia Hospital (Perugia), San Salvatore Hospital (L'Aquila), Galliera Hospital (Genova). 


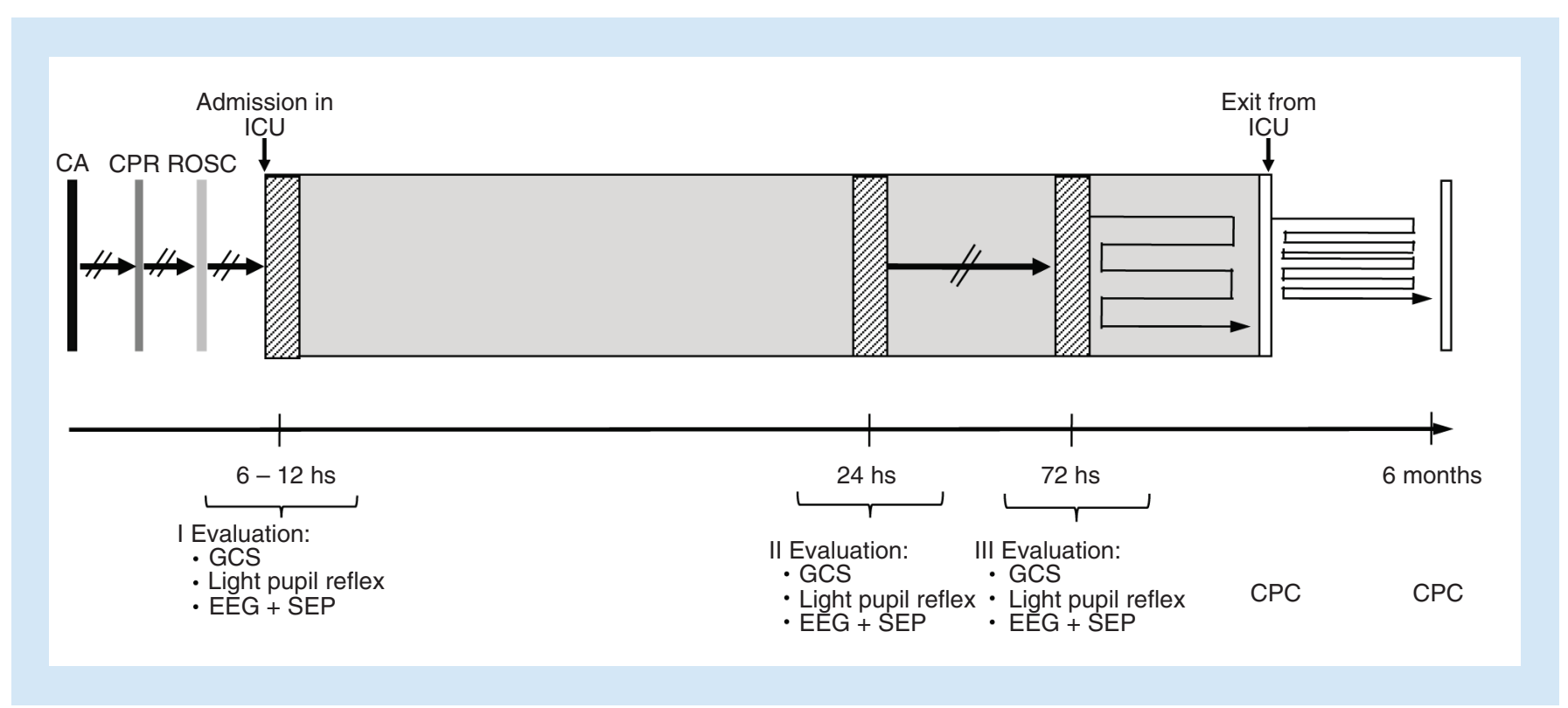

Figure 1. Course line of ProNeCA study.

CA: Cardiac arrest; CPC: Cerebral Performance Categories; CPR: Cardio-respiratory resuscitation; EEG: electroencefalogram; GCS: Glasgow Coma Scale; hs: Hours; ICU: Intensive care unit; SEP: Somatosensory evoked potentials.

\section{Ethical approval \& trial registration}

All the procedures will conform to the Good Clinical Practice standards and the study protocol was approved by the Comitato Etico Area Vasta Centro, Regione Toscana (Rif. N OSS.15.009). Written informed consent will be obtained from the patient's authorized representative prior to the performance of neurophysiological tests. Surviving cognitive patients will be asked for informed consent for participation and additional follow-up on long-term outcome. The study will be conducted in accordance with the Declaration of Helsinki.

\section{Participants \& recruitment}

Patients will be recruited among those admitted to the ICUs of all the participating centers to the study. The inclusion criteria will be: male and female patients between 18 and 90 years of age and comatose patients surviving after CA with a Glasgow Coma Scale $(\mathrm{GCS})<9$. The exclusion criteria will be: patients with surgical or traumatic causes of CA, patients showing contemporary presence of other neurological diseases (i.e., traumatic brain injury or brain infarction), patients with previous severe neurological diseases, patients with remote pathological anamnesis showing severe diseases with life expectancy less than 6 months, patients with previous severe disability, contemporary presence of confounding factors that hamper clinical evaluation (in particular the consciousness state), patients with contemporary absence of cortical response N20/P25 and lemniscal wave P14.

\section{Procedure}

A flow-chart outlining the study procedures is presented in Figure 1.

\section{Screening}

Study researchers will screen patients for eligibility. Once eligibility will be confirmed, written informed consent will be obtained from the patient's authorized representative. In line with the CONSORT guidelines $[11,12]$ we will record the number and reasons for any patients who will be excluded.

\section{EEG recording \& classification}

Standard 30 min EEG recordings will be organized into three time-frames relative to CA: 12, 24 or $72 \mathrm{~h}$. At least ten needles to 21 electrodes will be placed according to the international 10-20 system. Recordings will be acquired with a sampling rate of $128 \mathrm{~Hz}$. During reviewing digital filters (low-pass filter $=30$ to $70 \mathrm{~Hz}$; time constant $=0.1 \mathrm{or}$ $0.3 \mathrm{~s}$; notch filter $=50 \mathrm{~Hz})$ and sensitivity gain $(2$ to $10 \mu \mathrm{V} / \mathrm{mm}$ with a standard gain of $7 \mu \mathrm{V} / \mathrm{mm})$ will be adjusted 
according to interpretation needs. All the EEGs will be classified according to American Clinical Neurophysiology Society (ACNS) terminology [13]. Thus, the main patterns identified would be continuous, nearly continuous, discontinuous, low voltage (voltage $<20 \mu \mathrm{V}$ ), burst suppression, suppression (voltage $<10 \mu \mathrm{V}$ ), epileptiform discharges and isoelectric (voltage $<2 \mu \mathrm{V}$ ), although the original classification does not distinguish this last pattern from suppressed activity.

\section{SEP recording \& classification}

Electrical stimulation (pulse duration $0.2 \mathrm{~ms}$; stimulus rate $3 \mathrm{~Hz}$ ) of right and left median nerves at the wrist will be delivered by a bipolar surface electrode. In case of obstacles to the stimulus at the wrist, stimulation will be performed at the elbow. The reccomended intensity will be $4-5 \mathrm{~mA}$ above the motor threshold. In patients treated with muscle relaxants, the intensity of the stimulation will be estimated on the ERB potential amplitude. A 4-channel evoked potentials device will be used. Recording electrodes will be placed at the following locations to ensure recording of peripheral, spinal, bulbar and cortical component: Erb's point (referred to contralateral Erb's point) for N9 peak; spinous process CV 7 (referred to the anterior neck) for N13 peak; C3'/C4' (referred to ipsilateral mastoid) for P14 and C3'/C4' (referred to Fz) for N20/P25 complex. At least two averages of 300 stimuli will be acquired and superimposed to assess the reproducibility of SEP components. The analysis time will be $100 \mathrm{~ms}$ and bandwidth will be $5 \mathrm{~Hz}-3 \mathrm{kHz}$. Finally, in order to reduce noise levels $<0.25 \mathrm{uV}$ [14] administration of muscle relaxants and turning electric equipment off will be suggested.

N20 will be identified as a major negative peak with a latency of approximately $20 \mathrm{~ms}$ from the stimulus, P25 will be the major positive peak following N20. To define a pathological SEP, we will consider only N20/P25 complex peak-to-peak amplitude. N20/P25 amplitude will be classified as follows: Normal (N), Pathological (P) if N20/P25 amplitude will be lower than the limit of normality (the fifth percentile) of each participating center or the difference between the two sides will be greater than $50 \%$. Cortical SEP absence (A) will be considered reliable only when cervical N13 and Erb potential will be present. Taking into account the cortical responses of each hemisphere, we will obtain six SEP patterns: NN, NP, PP, AN, AP and AA. In the case of technically insufficient recording, SEPs will be defined as 'undeterminable' and the results will be excluded from the analysis. Recordings will be organized into the same time-frames in which EEG will be performed.

In case of unintended outcomes in patients with AA SEP pattern, the EEG pattern will be considered and then a post-hoc assessment will be performed in the following way: the original recording will be obtained and the traces will be mixed with at least ten other arbitrarily chosen SEP traces with defined AA pattern. All recordings will be assessed during an ad-hoc meeting by three blinded, experienced neurophysiologists recruited among those participating in the study but not involved in selection of SEP traces.

\section{Study process}

The study will consist of four stages:

1) Patient screening (Stage 0$)$

2) Neurological and instrumental evaluation (EEG and SEPs) at 12-24 and $72 \mathrm{~h}$ after CA (Stage 1)

3) Neurological evaluation at ICU discharge (Stage 2)

4) Six months of follow-up (Stage 3)

Stage 0: patients will be recruited among those admitted to the ICUs of all the participating centers to the study. Study researchers will screen patients for eligibility according to the inclusion and exclusion criteria. After patient enrollment, written informed consent will be obtained from the patient's authorized representative. Patient baseline demographic features will be collected as follows: first name, surname, age, gender, patient telephone number, hospital and ICU where patient is admitted, location of arrest (out-hospital/in-hospital), time at which CA occurred, if it was a witnessed arrest, CA duration, initial rhythm, CA causes and hypothermia treatment. These data will be collected according to the guidelines in literature [15].

Stage 1: all patients enrolled will undergo neurological evaluation. Clinical data concerning GCS score at ICU admission and pupillary reflex and diameter, taking in to account the possible use of cycloplegic drugs, will be collected. After clinical evaluation, neurophysiological tests (EEG and SEPs) will be performed.

Stage 2: neurological outcome evaluation will be assessed at the discharge from ICU, using CPC. 
Stage 3: for patients who will survive, neurological outcome evaluation will be assessed at least 6 months after CA by telephone interview conducted by an investigator for each center blinded to neurophysiological data. Subjects will leave the study at any time for any reason if they wish to do so without any consequences. Also, the investigators will be able to decide to withdraw a subject from the study for urgent medical reasons.

\section{Clinical management}

Each center will be able to follow its own treatment protocol concerning the use of the targeted temperature management (TTM): $34^{\circ} \mathrm{C}$ TTM, $36^{\circ} \mathrm{C}$ TTM or no TTM. Concerning sedative drugs, the use of low dosages of propofol (range $1-2 \mathrm{mg} / \mathrm{kg} / \mathrm{h}$ ) or midazolam (range $0.03-0.1 \mathrm{mg} / \mathrm{kg} / \mathrm{h}$ ) will be recommended because previous studies have shown that these neurosedation dosages did not alter EEG findings, in particular the continuity of the EEG [16,17], whereas, concerning SEPs, only mild changes in N20/P25 amplitude were observed [18-22]. Nonetheless, we will ask to report the sedative dosage used in the patient chart.

\section{Clinical protocol on outcome prediction}

All patients successfully resuscitated after CA will undergo EEG and SEPs at 12 and $24 \mathrm{~h}$ after cardiopulmonary resuscitation. The neurophysiological evaluation will be repeated in patients still comatose at $72 \mathrm{~h}$ after CA. After the first week, in agreement with the intensivists, patients with CPC 3 will be discharged toward a rehabilitation unit, whereas patients with CPC4 will be discharged toward a long-term care unit or toward a rehabilitation unit, according to the neurophysiological and clinical data. Except where patients with confirmed BD will be concerned, treatment will be continued in all patients and decisions to withdraw life support will not be included in the standards of care of participating centers to the study.

\section{Adverse events}

The neurophysiological tests (EEG and SEPs) that will be performed in this study will be the same that are included in the current clinical practice for comatose CA surviving patient management. Taking in the account our previous experience (we record about 700/year SEP and EEG with the same protocol in acute brain injury patients of several etiologies) we will expect no significant adverse events. Nonetheless, at each time-frame the study physicians will evaluate possible adverse events (AEs).

\section{Sample size calculation}

Our research hypothesis will be that combining the use of two diagnostic procedures, EEG and SEPs, a favorable or unfavorable prediction would be possible, with positive predictive value of good outcomes higher than $60 \%$, and a false-positive rate $(100 \%)$ for poor outcomes equal to 0 . Following this assumption and in agreement with the sample evaluation based on the asymptotic normal theory [23], in order to guarantee that the study will have a power equal to $80 \%$, with alpha of $5 \%$, it will be necessary to enrol 63 subjects with favourable outcomes and 63 subjects with unfavourable outcomes. The sample size calculation was adjusted for projected mortality without withdrawal of life-sustaining treatment (WLST), so, assuming that the proportion of surviving patients with good outcomes will be about $30 \%$ of the entire study population, it will be estimated that to observe at least 63 favourable events a minimum number of patients equal to 210 should be enrolled for each time-frame (12, 24 and $72 \mathrm{~h})$. As suggested by Schatzkin et al. [24], the sample size should be increased to 241 patients to have a $90 \%$ chance to observe at least 63 patients with favourable outcomes at the end of the study. Based on these assumptions a 95\% CI (using Wilson's method) of false positive rate (FPR) will be $0-3 \%$. The sample size of 240 participants for each recording time will be estimated assuming 24 months of accrual and a follow-up of 6 months after the last recruited patient. However, because of consideration of no funding for this study and human resources, the recruitment will be not prolonged beyond 30 months.

\section{Statistical analysis}

Baseline characteristics and raw distributions of the $\mathrm{CPC}$ score will be first presented in a descriptive way. Continuous variables will be presented as mean \pm standard deviation (SD) for normally distributed data and as median and interquartile ranges (IQR) for non-normally distributed data. Categorical variables will be presented as number and percent.

The accuracy of EEG and SEPs in predicting the poor and good outcomes in each time-frame will be first evaluated by plotting receiver operating characteristic (ROC) curves, which combine both the sensitivity and 
specificity achieved by the diagnostic test at several cut-off points [25]. We will express the performance of each neurophysiological test for predicting poor and good outcomes as the area under the curve (AUC) for ROC curves. Then we will determine sensitivity, specificity, positive predictive value (PPV), negative predictive value (NPV) and their 95\% confidence intervals (CI) collapsing the EEG and SEP patterns according to the cut-off selected from ROC curves. In particular, using a $2 \times 2$ table for each time-window group of patients we will analyze the following: the chance that patients with nonmalignant EEG patterns will have good outcomes; the chance that patients with malignant EEG patterns or SEP major alterations will have poor outcomes. The FPR and related CIs will be also calculated (FPR $=1-$ SPE). Missing data will be handled by multiple imputation method.

\section{Trial status \& timeline}

The ProNeCa study has been first presented at the National Congress of Società Italiana di Neurofisiologia Clinica held in Verona in May 2015, and then debated at an Investigation Meeting center organized in Florence on the $5^{\text {th }}$ of December 2015. The recruitment of the first ProNeCA patient started immediately after this meeting and participating centers have been gradually added, reaching the final number of 13 . As of January 2018, 400 patients had been recruited. We anticipate that the last 6 months follow-up will be performed in December 2018.

\section{Publication of trial results}

The results of the study will be published by the members of the coordinator centre on behalf of the ProNeCA study group. With ten or more patients recruited, the principal investigators of each participating center will be included in the main list of authors. The collaborators of each center will be included in the list of study participants. All the main local investigators will have the opportunity to review and comment on the manuscript before sending it.

\section{Discussion}

We present the protocol of a multicenter, prospective cohort study to investigate the ability of neurophysiological tests to predict both poor and good neurological outcomes in comatose patients at different times after CA. In particular, the focus of debate concerns the timing at which it is possible to make a reliable neurological prognostication, what kind of prognosis is possible (good, poor or both) and which neurophysiological tests allow this prognostication.

Previous studies have shown evidence of SEP and EEG utility for neurological outcome predictions [4-7] and they were included in the guidelines published in 2015 [9] that suggest a multimodal approach for both good and poor neurological outcomes, based on clinical, laboratory and instrumental data. Despite these recent suggestions, to date most papers, including those published after the drawing of this study, analyzed a single parameter (EEG or SEPs) at a time [4-6,10,26-28]. When the combination of these two tests is concerned, the major limitation is represented by the study protocol, having all the cases reported in each study collected only in a single center [8,29-33].

Thus, in order to provide a foundation for the use of EEG and SEPs for reliable prognostication of both poor and good outcomes in comatose patients from an early stage (within the first $12 \mathrm{~h}$ after $\mathrm{CA}$ ), prospective multicenter trials are needed.

To date, few studies $[4,5,34]$ performed a prospective evaluation, but in these papers only one test was analyzed at a time or only the poor neurological outcome was evaluated [8].

For this reason, through the present study we will try to overcome the limits of previous papers, performing a multicenter and prospective study evaluating the prognostic power of the association of EEG and SEPs for both good and poor neurological outcome predictions of CA comatose surviving patients at different times after CA.

The first aim of this paper is to confirm the preliminary results obtained in two previous retrospective, monocentric studies performed by our group and published after the drawing of this study [29,30], concerning the different time dependent prognostic features of these two neurophysiological tests and their complementary roles in prognostication of both poor and good outcomes. The strength of our study is represented by two main factors. Since both the neurophysiological tests require interpretation based on qualitative evaluation, standardization among the participant centers could be difficult. Nonetheless, concerning SEPs, the use of a classification that takes into account the cortical responses of each hemisphere should allow a homogeneous evaluation among the participating centers and will also provide a graduation of the severity of the alteration through the use of six different patterns (NN, NP, PP, AN, AP and AA) [35]. Concerning EEG, many previous studies tried to propose a grading system and definition of malignant and nonmalignant EEG patterns, which, however, complicate comparisons and meta-analyses. In addition, only a limited number of EEG patterns have been evaluated, so it is reasonable to assume that the interpreting EEG-specialists could have been biased by other EEG features. Thus, a standardized 
terminology evaluating all important features would be preferable to assess which individual features of the EEG are the most predictive. The ACNS proposed a revised version of a standardized critical care EEG terminology [13], characterizing both rhythmic and periodic patterns as well as background activity. The use of this terminology, as in our case, should allow collaborative, multicenter studies and maximize inter-rater reliability.

A second main topic concerns the natural history of patients surviving after CA, that to date is still unknown. Its evaluation is mainly influenced by WLST, a management widely used in the clinical practice. In fact, WLST could affect the possibility of survival, since it represents a common cause of hospital death after CA when a perceived poor neurological prognosis is concerned. Elmer et al. [36] showed that $26 \%$ of the patients who underwent early (before $72 \mathrm{~h}$ after CA) WLST might have survived, had life-sustaining therapy not been withdrawn, and that $64 \%$ of them might have had a functionally favorable survival. Failure to control for the effects of WLST may significantly bias the results of studies of CA. Actually, the risk of self-fulfilling prophecy and its management are reported as a limitation in most of the papers dealing with prognostication of comatose patients surviving after CA. In our clinical protocol life-sustaining treatments are never suspended, regardless of poor outcomes suggested by clinical or instrumental findings. This allows us as consequence to observe the 'real natural evolution' of patients surviving after CA. This represents a strength of our study, because it will allow us to verify the reliability of our early prediction on a clinical context where the risk of self-fulfilling prophecy is limited, and this, hopefully, will avoid any self-fulfilling prophecy bias, in contrast to the majority of previous studies. Finally, it will allow us to investigate EEG and SEP pattern changes according to different time-frames after CA.

Our protocol, however, has some limitations. First of all, patients evaluated at 12 and $24 \mathrm{~h}$ after CA will usually still be treated with sedative drugs. We will recommend low dosages of propofol or midazolam: with this condition EEG usually remains continuous $[16,17]$ and only minor changes of cortical SEP amplitude or latency are usually observed both in normal subjects [20-22] and patients with acute brain damage of several etiologies and with different types of cortical SEP patterns [18,19]. Moreover, as each center will be left free to follow its treatment protocol, not all the recruited patients will be subjected to TTM. Nonetheless, concerning SEPs, a previous study by our group showed that in patients at $34^{\circ} \mathrm{C}$ only significant changes of the CCT and not of the N20/P25 amplitude are observed [37]. Concerning EEG instead, it is well known by the literature that at $34^{\circ} \mathrm{C}$ only shifting in EEG frequencies is induced, whereas no significant changes in the continuity of the background activity are observed [38]. Nonetheless, even if we can consider that temperature will not significantly alter SEP and EEG findings, a subgroup analysis will be performed to determine a possible effect of TTM treatment on the prognostic value of the EEG and SEP patterns. We limited evaluation to the N20/P25 cortical complex. Later components of SEPs have been suggested as useful indicator of a mild HIE, then useful to establish a good prognosis. The utility of later SEP components have been investigated previously but with no homogeneous results. That's why we preferred instead to investigate two neurophysiological tests that could give us complementary prognostic information according to the time after CA. Finally, we are aware that neurophysiological tests are only some of the predictors proposed by recent guidelines [39-41] for neurological outcome evaluation of CA patients. A recent retrospective, monocentric study [31,32], published after the drawing of this study, analyzed the prognostic power of the association of these neurophysiological tests (EEG and SEPs) with neuroimaging (brain computed tomography CT), performed in a single subject, showing an increase in the sensitivity of patients correctly identified early (within $24 \mathrm{~h}$ after CA) with poor outcomes compared with the use of a single predictor at a time. In our original protocol design, we have not included brain CT in patient's data chart collection. However, considering the encouraging results of this previous study, we will ask all the participating centers for the availability of early neuroimaging data for a possible subgroup analysis.

In conclusion, ProNeCA will be the first multicenter prospective study evaluating the prognostic power of the association of two neurophysiological tests, performed in a single subject, for the prediction of both poor and good neurological outcomes of comatose CA patients at different times after CA in a cohort of patients in which WLST is not a standard of care. This last feature should allow verification of the prognostic reliability of the neurophysiological tests, avoiding the risk of self-fulfilling prophecy.

\section{Financial \& competing interests disclosure}

The authors have no relevant affiliations or financial involvement with any organization or entity with a financial interest in or financial conflict with the subject matter or materials discussed in the manuscript. This includes employment, consultancies, honoraria, stock ownership or options, expert testimony, grants or patents received or pending, or royalties.

No writing assistance was utilized in the production of this manuscript. 
Open access

This work is licensed under the Creative Commons Attribution-NonCommercial-NoDerivatives 4.0 Unported License. To view a copy of this license, visit: http://creativecommons.org/licenses/by-nc-nd/4.0/

\section{Summary points}

- Postanoxic encephalopathy is a severe and frequent neurological complication of successful cardiopulmonary resuscitation, with a challenging prognosis.

- Neurological prognosis of patients with hypoxic-ischemic encephalopathy (HIE) after cardiac arrest (CA) requires a multimodal approach.

- Most of previous studies were retrospective and/or evaluating only one parameter at a time as neurological predictor.

- According to the literature, neurophysiological tests (electroencephalogram [EEG] and somatosensory evoked potentials [SEPs]) are reliable prognostic indicators for neurological outcomes.

- This is a prospective, Italian multicenter study (ProNeCA) evaluating neurological prognosis of patients with hypoxic-ischemic-encephalopathy after CA, using the combination of EEG and SEPs.

- EEG and SEPs will be recorded in the same patients at different time-frames after cardiac arrest (12-24 and $72 \mathrm{~h}$ ).

- The principal aim will be to evaluate the prognostic power of EEG performed in comatose patients within the first $12 \mathrm{~h}$ after CA for good outcome prediction and to evaluate its prognostic power for the poor outcome prediction when performed at 24 and $72 \mathrm{~h}$ after CA.

- The second aim will be to evaluate if the combination of EEG and SEPs will enable us to correctly identify a greater number of patients with both poor and good outcomes (when performed within the first $12 \mathrm{~h}$ ) and with poor outcomes (when performed after $72 \mathrm{~h}$ ) compared with the use of only a single test.

\section{References}

Papers of special note have been highlighted as: $\bullet$ of interest; $\bullet \bullet$ of considerable interest

1. Gorjup V, Radsel P, Kocjancic ST, Erzen D, Noc M. Acute ST-elevation myocardial infarction after successful cardiopulmonary resuscitation. Resuscitation 72, 379-385 (2007).

2. Hosmane VR, Mustafa NG, Reddy VK et al. Survival and neurologic recovery in patients with ST-segment elevation myocardial infarction resuscitated from cardiac arrest. J. Am. Coll. Cardiol. 53, 409-415 (2009).

3. Scarpino M, Grippo A, Lanzo G, Lolli F. The burden of clinical neurophysiology for the neurological prognosis of coma. Future Neurol. 13, 127-129 (2018a).

4. Bowes A, Binnekade JM, Kuiper MA, Bosch FH, Zandstra DF, Toornvlirt AC. Prognosis of coma after therapeutic hypothermia: a prospective cohort study. Ann. Neurol. 71, 206-212 (2012).

- First Multicenter prospective study using somatosensory evoked potentials as neurological predictor.

5. Cloostermans MC, van Meulen FB, Eertman CJ, Horn HW, van Putten MJ. Continuous electroencephalography monitoring for early prediction of neurological outcome in post-anoxic patients, after cardiac arrest: a prospective cohort study. Crit. Care Med. 40, 2867-2875 (2012).

6. Crepeau AZ, Rabinstein AA, Fugate JE. Continuous EEG in therapeutic hypothermia after cardiac arrest. Prognostic and clinical value. Neurology 80, 339-344 (2013).

7. Wijdicks EF, Hijdra A, Young GB, Bassetti GL, Wiebe S. Quality Standards Subcommittee of the American Academy of Neurology. Practice parameter: prediction of outcome in comatose survivors after cardiopulmonary resuscitation (an evidence-based review): report of the Quality Standards Subcommittee of the American Academy of Neurology. Neurology 67, $203-210$ (2006).

-• First guidelines about poor neurological prognosis of hypoxic-ischemic-encephalopathy (HIE) patients.

8. Oddo M, Rossetti AO. Early multimodal outcome prediction after cardiac arrest in patients treated with hypothermia. Crit. Care 42, 1340-1347 (2014).

9. Nolan JP, Soar J, Cariou A. European Resuscitation Council and European Society of Intensive Care Medicine Guidelines for post-resuscitation care 2015: section 5 of the European Resuscitation Council Guidelines for resuscitation 2015. Resuscitation 95 , 202-222 (2015).

-. Guidelines about multimodal approach for poor neurological prognosis of HIE patients.

10. Rundgren M, Westhall E, Cronberg T, Rosen I, Friberg H. Continuous amplitude-integrated electroencephalogram predicts outcome in hypothermia-treated cardiac arrest patients. Crit. Care Med. 38, 1838-1844 (2010).

11. Moher D, Schulz KF, Altman DG. Group C. The CONSORT statement:revised recommendations for improving the quality of reports of parallel-group randomized trials. Lancet 357, 1191-1194 (2001). 
12. Schulz KF, Altman DG, Moher D. CONSORT 2010 statement: update guidelines for reporting parallel group randomized trials. Ann. Intern. Med. 152, 726-732 (2010).

13. Hirsch LJ, La Roche SM, Gaspard N et al. American Clinical Neurophysiology Society's Standardized Critical Care EEG Terminology: 2012 version. J. Clin. Neurophysiol. 30, 1-27 (2013).

- Standardized electroencephalogram terminology in comatose patients.

14. Zandbergen EG, Hijdra A, de Haan RJ et al. Inter-observer variation in the interpretation of SEPs in anoxic-ischaemic coma. Clin. Neurophysiol. 117, 1529-1535 (2006).

15. Jacobs I, Nadkarni V; the ILCOR Task Force on Cardiac Arrest and Cardiopulmonary Resuscitation Outcomes. Cardiac Arrest and Cardiopulmonary Resuscitation Outcome. Reports Update and Simplification of the Utstein Templates for Resuscitation Registries A Statement for Healthcare Professionals From a Task Force of the International Liaison Committee on Reuscitation (American Heart Association, European Resuscitation Council, Australian Resuscitation Council, New Zeland Resuscitation Council, Heart and Stroke Foundation of Canada, InterAmerica Heart Foundation, Resuscitation Councils of Southern Africa). Circulation 110, 3385-3397 (2004).

16. San-Juan D, Chiappa KH, Cole AJ. Propofol and the Electroencephalogram. Clin. Neurophysiol. 121, 998-1006 (2010).

17. Veselis RA, Reinsel R, Marino P. The effects of midazolam on the EEG during sedation of critically ill patients. Anaesthesia 48, 463-470 (1993).

18. Amantini A, Fossi S, Grippo A et al. Continuous EEG-SEP monitoring in severe brain injury. Neurophysiol. Clin. 39, 85-93 (2009).

19. Fossi S, Amantini A, Grippo A et al. Continuous EEG-SEP monitoring of severely brain injuredpatients in NICU: methods and feasibility. Neurophysiol. Clin. 36, 195-205 (2006).

20. Koht A, Schutz W, Schmidt G, Schramm J, Watanabe E. Effects of etomidate, midazolam, and thiopental on median nerve somatosensory evoked potentials and the additive effects of fentanyl and nitrous oxide. Anesth. Analg. 67, 435-441 (1988).

21. Scheepstra GL, de Lange JJ, Booij LH, Ros HH. Median nerve evoked potentials during propofolanaesthesia. Br. J. Anaesth. 62, 92-94 (1989).

22. Sloan TB, Fugina ML, Toleikis JR. Effects of midazolam on median nerve somatosensory evoked potentials. Br. J. Anaesth. 64, 590-593 (1990).

23. Pepe MS. The Statistical Evaluation of Medical Tests for Classification and Prediction. Oxford University Press, UK (2003).

24. Schatzkin A, Connor RJ, Taylor PR, Bunnag B. Comparing new and old screening tests when a reference procedures cannot be performed on all screenes. Am. Jo. Epidemiol. 125, 672-678 (1987).

25. Fletcher RH, Fletcher SW, Wagner EH. (Eds.) Clinical Epidemiology: The Essentials. Lippincott Williams and Wilkins, MA, USA (1996).

26. Spalletti M, Carrai R, Scarpino M et al. Single electroencephalographic patterns as specific and time-dependent indicators of good and poor out come after cardiac arrest. Clin. Neurophysiol. 127, 2610-2617 (2016).

27. Scarpino M, Lanzo G, Lolli F et al. Predictive patterns of sensory evoked potentials in comatose brain injured patients evolving to brain death. Neurophysiol. Clin. 47, 19-29 (2017a).

28. Scarpino M, Lanzo G, Lolli F et al. Is brain computed tomography combined with somatosensory evoked potentials useful in the prediction of brain death after cardiac arrest? Neurophysiol. Clin. 47, 327-335 (2017b).

29. Carrai R, Grippo A, Scarpino M et al. Time-dependent and independent neurophysiological indicators of prognosis in post-anoxic coma subjects treated by therapeutic hypothermia. Minerva Anest. 82, 940-949 (2016).

30. Grippo A, Carrai R, Scarpino $\mathrm{M}$ et al. Neurophysiological prediction of neurological good and poor out come in post-anoxic coma. Acta Neurol. Scand. 135, 641-648 (2017).

- The first attempt to combine EEG and SEPs for good and poor neurological prognosis of HIE patients at different times after CA.

31. Scarpino M, Lanzo G, Lolli F et al. Neurophysiological and neuroradiological multimodal approach for early poor outcome prediction after cardiac arrest. Resuscitation 129, 114-120 (2018b).

- The first attempt to combine EEG, SEPs and neuroimaging for early poor neurological prognosis of HIE patients.

32. Scarpino M, Lanzo G, Lolli F et al. Data on multimodal approach for early poor outcome (Cerebral Performance Categories 3-5) prediction after cardiac arrest. Data Brief 19, 704-711 (2018c).

33. Carrai R, Scarpino M, Lolli F et al. Early SEPs' amplitude reduction is reliable for poor-outcome prediction after cardiac arrest? Acta Neurol. Scand. 139(2), 158-165 (2018).

34. Westhall E, Rossetti AO, van Rootselaar AF et al. TTM-trial investigators. Standardized EEG interpretation accurately predicts prognosis after cardiacarrest. Neurology 86, 1482-1490 (2016).

35. Amantini A, Grippo A, Fossi S et al. Prediction of "awakening" and outcome in prolonged acute coma from severe traumatic brain injury: evidence for validity of short latency SEPs. Clin. Neurophysiol. 116, 229-235 (2005).

36. Elmer J, Torres C, Aufderheide TP et al. the Resuscitation Outcomes Consortium. Association of early withdrawal of life-sustaining therapy for perceived neurological prognosis with mortality after cardiac arrest. Resuscitation 102, 127-135 (2016). 
37. Grippo A, Carrai R, Fossi $\mathrm{S}$ et al. Absent SEP during therapeutic hypothermia did not reappear after re-warming in comatose patients following cardiac arrest. Minerva Anestesiol. 79, 360-369 (2013).

38. Deboer T. Brain temperature dependent changes in the electroencephalogram power spectrum of humans and animals. J. Sleep Res. 7 , 254-262 (1998).

39. Taccone FS, Baar I, De Deyne C, Druwe P, Legros B, Meyfroidt G. Neuroprognostication after adult cardiac arrest treated with targeted temperature management: task force for Belgian recommedations. Acta Neuro. Belg. 117, 3-15 (2017).

-. Guidelines about multimodal approach for poor and good neurological prognosis of HIE patients.

40. Sandroni C, D’Arrigo S. Neurologic prognostication: neurologic examination and current guidelines. Semin. Neurol. 37, 40-47 (2017).

- A reappraisal of neurological prognostication in patients surviving to cardiac arrest.

41. Thomke F. Assessing prognosis following cardiopulmonary resuscitation and therapeutic hypothermia - a critical discussion of recent studies. Dtsch Arztebl. Int. 110, 137-143 (2013). 